\title{
Time Rate of Blood Pressure Variation Is Associated With Endothelial Function in Patients With Metabolic Syndrome
}

\author{
Yanping RuAN, ${ }^{1} \mathrm{MD}$, Wanlin WeI, ${ }^{2} \mathrm{MD}$, Jianhua YAN, ${ }^{3} \mathrm{MD}$, Lixian Sun,${ }^{4} \mathrm{MD}$, Hui Lian, ${ }^{1} \mathrm{MD}$, \\ Xiaoyi Zhao, ${ }^{1}$ MD, Ruijuan Liang,,${ }^{1} \mathrm{MD}$, Liu Xiaole, ${ }^{1} \mathrm{MD}$, and Zhongjie Fan, ${ }^{1} \mathrm{MD}$
}

\section{SUMMARY}

The time rate of blood pressure (BP) variation indicates the speed of BP fluctuations. Previous studies have demonstrated that the time rate of BP variation was associated with target organ damage. However, the association between time rate of $\mathrm{BP}$ variation and endothelial function has not been evaluated.

24-hour ambulatory blood pressure monitoring (ABPM) was performed in 61 patients with metabolic syndrome. Time rate of BP variation was calculated from BP recordings of ABPM. Endothelial function was assessed using reactive hyperemia-peripheral arterial tonometry index (RHI) by EndoPat2000. Multiple linear regression models were used to detect the association between time rate of $\mathrm{BP}$ variation and RHI.

Among all the subjects $(n=61)$, the multiple linear regression models revealed that the daytime rate of systolic blood pressure (SBP) variation was independently associated with $\mathrm{RHI}(\beta=-0.334, P=0.008)$. A $0.1 \mathrm{mmHg} /$ minute increase in the daytime rate of SBP variation correlated with a decline of 0.20 in RHI. The same effect was also found in the subjects with eGFR $\geq 60 \mathrm{~mL}$ ( $\left(\right.$ minute* $1.73 \mathrm{~m}^{2}$ ). A greater association was found in those who were not taking a statin, $\beta$-blocker, ACEI/ARB, or diuretic and those without diabetes compared with those with any antihypertensive medication or with diabetes. Other ambulatory blood pressure parameters and central hemodynamics were not found to be associated with RHI.

Our findings have shown that the daytime rate of SBP variation was associated with endothelial function in patients with metabolic syndrome, independent of other BP parameters and central hemodynamics. (Int Heart J 2016; 57: 226233)

Key words: RHI, Ambulatory blood pressure monitoring, Target organ damage, Blood pressure variability

$\mathrm{A}$ mbulatory blood pressure monitoring (ABPM) is widely used in patients with prehypertension and hypertension, and its use is gradually increasing. Many studies have suggested that systolic blood pressure (BP), diastolic BP, and the degree of BP variability (BPV) calculated from $\mathrm{ABPM}$ are associated with target organ damage, cardiovascular disease risk, and long-term prognosis. ${ }^{1,2)}$ Mancia, et al described a new BP phenomenon called time rate of BP variation, which is a measure of the speed of BP fluctuations derived from a computerized analysis of ABPM data. ${ }^{3)}$ Current evidence has suggested that the time rate of $\mathrm{BP}$ variation was associated with target organ damage, such as increased common carotid artery intima-media thickness, ${ }^{4,5)}$ increased left ventricular mass, ${ }^{6}$ impaired renal function, ${ }^{7)}$ and coronary atherosclerosis. ${ }^{8)}$

Endothelial dysfunction is one of the important pathophysiological processes of the early stage in patients with metabolic syndrome (MetS), and is an early manifestation before atherosclerotic plaque detected by angiography or ultrasound, ${ }^{9)}$ and is a sign of poor cardiovascular prognosis. ${ }^{10)}$ Early detection of endothelial dysfunction has attracted more and more attention in clinical practice. Reactive hyperemia-peripheral arterial tonometry index (RHI) is a non-invasive, automatic, and quantitative digital method to measure hyperemia response as described by Kuvin, et al. ${ }^{11)}$ The Framingham Heart Study has reported that RHI, as an index of endothelial function, was negatively correlated with cardiovascular risk factors. ${ }^{12)}$

The faster the BP changes in a given period, the greater the shear stress the vessel walls suffer from, which results in endothelial dysfunction initially, followed by the advancement of atherosclerosis. ${ }^{13)}$ However, the association between the rate of BP variation and endothelial function has been rarely evaluated. The present cross-sectional study aimed to investigate the relative importance of time rate of BP variation and endothelial function, assessed using RHI in patients with MetS. In addition, central hemodynamics was independently associated with

From the ${ }^{1}$ Department of Cardiology, Peking Union Medical College Hospital, Peking Union Medical College and Chinese Academy of Medical Sciences, ${ }^{2}$ Department of Cardiology, General Hospital of Beijing Military Command, Beijing, ${ }^{3}$ Division of Cardiovascular Medicine, The Xinhua Hospital Affiliated to Shanghai Jiaotong University School of Medicine, ${ }^{4}$ Division of Cardiovascular Medicine, The Affiliated Hospital of Chengde Medical College, Chengde Medical College, Chengde, Hebei, China.

Address for correspondence: Zhongjie Fan, MD, Department of Cardiology, Peking Union Medical College Hospital, No. 1 Shuai fu yuan, Dongcheng District, Beijing, China. E-mail:Fan@pumch.cn

Received for publication August 14, 2015. Revised and accepted October 30, 2015.

Released in advance online on J-STAGE March 11, 2016.

All rights reserved by the International Heart Journal Association. 
the development of target organ damage and prognosis of cardiovascular diseases. ${ }^{14)}$ The secondary goal of the study was to evaluate its association with RHI.

\section{MeThodS}

Participants and design: Subjects with MetS $(n=61)$ were recruited from clinics affiliated with Peking Union Medical College Hospital (PUMCH) between February and July 2013. Eligibility criteria included non-smoking adults between 35-75 years of age living in a non-smoking home in Beijing. MetS was defined by International Diabetes Federation (IDF) criteria specific for Asians, with waist circumference $\geq 90 \mathrm{~cm}$ in males and $\geq 80 \mathrm{~cm}$ in females plus any two of the following 4 factors: 1) triglyceride (TG) level $>1.7 \mathrm{mmol} / \mathrm{L}$ or taking lipid-lowering medication; 2) high density lipoprotein cholesterol (HDLC) $<1.04 \mathrm{mmol} / \mathrm{L}$ in males and $<1.29 \mathrm{mmol} / \mathrm{L}$ in females; 3) $\mathrm{BP} \geq 130 / 85 \mathrm{mmHg}$ or taking antihypertensive therapy; and 4) fasting blood glucose $\geq 5.6 \mathrm{mmol} / \mathrm{L}$ or previously diagnosed type 2 diabetes mellitus. Exclusion criteria included intake of drugs that may alter endothelial function (eg, antioxidants, multi-vitamins, folic acid, fish oil supplementation, L-arginine). The research protocol was approved by the Institutional Review Board (IRB) at PUMCH and all subjects signed a written informed consent form.

Ambulatory blood pressure monitoring (ABPM) and data analyses: All patients underwent 24-hour ABPM on a normal working day. Ambulatory BP was recorded using an oscillometric Spacelabs 90207 (Spacelabs Healthcare ABP Monitors; 90207, WA, USA). They were instructed to act and work as usual and to keep their non-dominant arm still and relaxed during measurements. BP readings were automatically obtained every 20 minutes during the day (6:00-22:00) and every 30 minutes during the night (22:00-6:00). Meanwhile, 24-hour activity log was recorded every 20 or 30 minutes accordingly.

The mean 24-hour, daytime, nighttime BP (systolic BP (SBP) and diastolic BP (DBP)), and blood pressure variability, such as standard deviation (SD) and coefficient of variation (CV) of SBP and DBP for each patient were calculated. Meanwhile, the rate of BP variation during 24-hour, daytime, and nighttime were calculated using EXCEL (Microsoft Excel 2010, www.microsoft.com). The time rate of BP variation was defined as the first derivative of the BP values against time. This parameter, derived from ABPM data analysis, aimed to measure how fast or how slow BP changes. Given 2 SBP readings, $\mathrm{SBP}_{i}$ and $\mathrm{SBP}_{i+1}$ at time $\mathrm{t}_{i}$ and $\mathrm{t}_{i+1}$ respectively, the rate of SBP change is defined as: ${ }^{4)}$

$$
\text { (1) } \mathrm{r}_{\mathrm{i}}=\frac{\mathrm{SBP}_{\mathrm{i}+1}-\mathrm{SBP}_{\mathrm{i}}}{t_{\mathrm{i}+1}-t_{\mathrm{i}}}
$$

If there are $\mathrm{N}$ recordings of SBP during the 24-hour period, the mean rate of SBP change during this period can be calculated as follows: ${ }^{4)}$

$$
\text { (2) } \mathrm{R}=\frac{\sum_{\mathrm{i}-1}^{\mathrm{N}-1}\left|r_{i}\right|}{\mathrm{N}-1}
$$

Endothelial function: Peripheral endothelial function was assessed using an EndoPat2000 (Itamar Medical; 38,900, Caesarea, Israel; http://www.itamar-medical.com/), a semi-automat- ed FDA-approved system, which was utilized to measure RHI as the primary outcome. According to the manufacturer's protocol, a BP cuff was placed on one upper arm, while the contralateral arm served as a control. Endo-PAT bio-sensors are placed on the index fingers of both arms. The test takes 15 minutes to complete, is very easy to perform, and is both operator and interpreter independent. Thermo-neutral, quiet surroundings are recommended. Endo-PAT quantifies the endothelium-mediated changes in vascular tone, elicited by a 5-minute occlusion of the brachial artery (using a standard blood pressure cuff). When the cuff is released, the surge of blood flow causes an endothelium-dependent flow mediated dilatation. The dilatation, manifested as reactive hyperemia, is captured by the Endo-PAT as an increase in the PAT signal amplitude. A post-occlusion to pre-occlusion ratio is calculated by the Endo-PAT software (Endo-PAT 2000 software version 3.0.4), expressed as RHI. RHI > 1.67 represents normal endothelial function, while RHI $\leq 1.67$ means abnormal endothelial function.

Pulse Wave Analysis (PWA): PWA was estimated with a SphygmoCor CPV non-invasive central blood pressure assessment device (AtCor Medical; NSW, Australia; http://atcormedical.com/index.html). In addition to aortic blood pressure, there are 3 indices [aortic pulse pressure (APP), augmentation pressure (AP), and augmentation index (AIx)] which reflect the stiffness of arteries.

Determination of biochemical indicators: All patients fasted for 8 hours or longer, and venous blood samples were taken in the morning to determine triglycerides (TG), total cholesterol (TC), low-density lipoprotein cholesterol (LDL-C), high-density lipoprotein cholesterol (HDL-C), blood glucose, and glycosylated hemoglobin $(\mathrm{HbA} 1 \mathrm{c})$. The indicators above were measured by technicians at the clinical lab in PMUCH.

Statistical analyses: Statistical comparisons were performed between females and males, including baseline characteristics, ABPM parameters, and biochemical measurements. Dichotomous variables were compared using the chi-square test and continuous variables using one-way analysis of variance (ANOVA). Simple and multiple linear regression analyses were performed to evaluate the factors independently associated with RHI. In the initial simple regression analyses, a threshold of $P<0.1$ was used to identify candidate variables for inclusion in the final model (because of the risk of type II error as a result of low statistical power in such an analysis). The multiple regression analyses were performed using a combination of stepwise and forward selection procedures, which is preferable to backward selection in datasets in which concerns about multi-collinearity are raised. The activity scale was also adjusted in the analysis. The unstandardized coefficients (B) and standardized coefficients $(\beta)$ were used to express the magnitude of the association. Sensitivity analysis was performed in the association between RHI and daytime rate of SBP variation grouped by medication use or diabetes. Statistical significance was achieved with a two-tailed value of $P<$ 0.05. The Statistical Package for Social Science, version 17 for Windows (SPSS17.0), was used for statistical analyses.

\section{RESULTS}

Sixty-one subjects (mean age $61.4 \pm 9.0$ years old) with 
MetS were enrolled, $42.6 \%$ of which were male. The proportions of hypertension, diabetes, and dyslipidemia among the study population were $82 \%, 26 \%$, and $77 \%$, respectively. Patients with diabetes were treated with acarbose, and had wellcontrolled blood glucose. The general characteristics of all subjects are presented in Table I. There were 4 subjects with eGFR $<60 \mathrm{~mL} /\left(\right.$ minute* $\left.1.73 \mathrm{~m}^{2}\right), 2$ of whom had albuminuria. Ambulatory BP parameters are presented in Table II. Time rate of $\mathrm{BP}$ variation was the highest in the morning surge and lowest during the nighttime $(P<0.05)$.

There was no significant difference in RHI between females and males. However, the 24-hour, daytime rate of SBP variation, and rate of SBP variation during the morning surge in females were higher than in males (all $P<0.05$ ), as were aortic SBP, APP, AP, and AIx (all $P<0.05$ ) (Supplemental Table I).

The associations of baseline characteristics, ABPM parameters, and PWA parameters with RHI were investigated using simple and multiple linear regression models. In the simple linear regression analysis presented in Table III, there were several variables associated with RHI as follows according to a threshold of $P<0.1$ : age, glucose, HbA1c, triglycerides, use of statins, 24-hour rate of SBP variation, daytime rate of SBP variation, average rate of SBP variation during morning surge, SD of daytime SBP, and CV of daytime SBP, but not central hemodynamics. There were also significant associations between RHI and these variables, including 24-hour rate of SBP variation $(\beta=-2.673, P=0.022)$, daytime rate of SBP variation $(\beta=-2.281, P=0.016)$, rate of SBP variation during morning BP surge $(\beta=-1.273, P=0.034)$, and glucose $(\beta=$ $-0.134, P=0.018)$ even after adjusting for gender, age, BMI, disease status, 24-hour BP, total cholesterol, triglycerides, and other medications (Supplemental Table II). Pearson correlation analysis was performed to evaluate the collinearity of independent variables added in the multiple regression models in Supplemental Table III. There were two pairs of significant correlated variables with a correlation coefficient greater than 0.80: 24-hour and daytime rate of SBP variation (correlation coefficient $=0.949$ ), and SD of daytime SBP and CV of daytime SBP (correlation coefficient $=0.925$ ). In the simple linear regression analyses, RHI was more strongly associated with daytime rate of SBP variation $(\beta=-0.323)$ than 24 -hour rate of SBP variation $(\beta=-0.302)$, and with $\mathrm{SD}$ of daytime $\operatorname{SBP}(\beta=$ $-0.244)$ than $\mathrm{CV}$ of daytime $\operatorname{SBP}(\beta=-0.219)$, and therefore only daytime rate of SBP variation and SD of daytime SBP were offered in the multiple regression models (Table III). The multiple regression analysis results shown in Table IV indicated that daytime rate of SBP variation was independently associated with RHI $(\beta=-0.334, P=0.008)$ with tolerance and Variance Inflation Factor (VIF) of 0.998 and 1, respectively. A $0.1 \mathrm{mmHg} /$ minute increase in the daytime rate of SBP variation correlated with a decline of 0.20 in RHI. Moreover, the multiple regression analyses using the backward and forward selection procedure were repeatedly performed so as to confirm the robustness of the relationship between daytime rate of SBP variation and RHI $(\beta=-0.334$ in forward procedure or $\beta$ $=-0.248$ in backward procedure) (Supplemental Table IV).

Whether or not the association between RHI and time rate of BP variation was modified by medication use was also investigated in the present study (Table V), which showed that a greater association between daytime rate of SBP variation
Table I. General Characteristics of Subjects With Metabolic Syndrome

\begin{tabular}{|c|c|}
\hline Variable & $n=61$ \\
\hline Age (years) & $61.4(9.0)$ \\
\hline Male $(\%)$ & $26(42.6)$ \\
\hline Body mass index $(\mathrm{BMI})\left(\mathrm{kg} / \mathrm{m}^{2}\right)$ & $26.0(2.8)$ \\
\hline Hypertension (\%) & $50(82)$ \\
\hline Diabetes (\%) & $16(26)$ \\
\hline Dyslipidemia (\%) & $47(77)$ \\
\hline \multicolumn{2}{|l|}{ Medication use } \\
\hline Aspirin (\%) & $44(72)$ \\
\hline Statins $(\%)$ & $34(56)$ \\
\hline$\beta$-Blocker $(\%)$ & $22(36)$ \\
\hline Calcium channel blocker $(\%)$ & $32(53)$ \\
\hline Angiotensin-converting enzyme inhibitor (\%) & $4(7)$ \\
\hline Angiotensin receptor blocker (\%) & $23(38)$ \\
\hline Diuretics (\%) & $12(20)$ \\
\hline Acarbose $(\%)$ & $16(26)$ \\
\hline \multicolumn{2}{|l|}{ Endothelial function } \\
\hline RHI & $2.08(0.53)$ \\
\hline \multicolumn{2}{|l|}{ PWA } \\
\hline Aortic SBP (mmHg) & $119.3(13.9)$ \\
\hline Aortic DBP (mmHg) & $78.3(9.9)$ \\
\hline Augmentation pressure (AP) (mmHg) & $14.4(6.6)$ \\
\hline Augmentation index(AIx) & $33.9(8.4)$ \\
\hline Aortic pulse pressure(APP) (mmHg) & $41.0(12.0)$ \\
\hline \multicolumn{2}{|l|}{ Biochemical indicators } \\
\hline Glucose $(\mathrm{mmol} / \mathrm{L})$ & $5.72(1.63)$ \\
\hline $\operatorname{HbA1c}(\%)$ & $6.25(1.00)$ \\
\hline Total cholesterol (mmol/L) & $4.64(1.13)$ \\
\hline Triglycerides $(\mathrm{mmol} / \mathrm{L})$ & $1.58(0.78)$ \\
\hline HDL-C (mmol/L) & $1.21(0.26)$ \\
\hline LDL-C (mmol/L) & $2.65(0.78)$ \\
\hline ApoA I (g/L) & $1.57(0.27)$ \\
\hline ApoB (g/L) & $0.88(0.23)$ \\
\hline Urea & $6.97(10.2)$ \\
\hline Creatinine & $70.9(21.5)$ \\
\hline eGFR(CKD-EPI) (mL/(minute*1.73 m²)) & $98.8(28.6)$ \\
\hline $\mathrm{eGFR}<60 \mathrm{~mL} /\left(\right.$ minute $\left.* 1.73 \mathrm{~m}^{2}\right)(\%)$ & $4(7)$ \\
\hline eGFR $\geq 60 \mathrm{~mL} /\left(\right.$ minute* $\left.1.73 \mathrm{~m}^{2}\right)(\%)$ & $57(93)$ \\
\hline \multicolumn{2}{|l|}{ Albuminuria } \\
\hline Positive (\%) & $2(3)$ \\
\hline Negative (\%) & $59(97)$ \\
\hline
\end{tabular}

Noncontinuous variables are given as percentages. Continuous variables are presented as mean with standard deviation (SD). RHI indicates reactive hyperemia index; PWA, pulse wave analysis; PWV, pulse wave velocity; SBP, systolic blood pressure; DBP, diastolic blood pressure; HbA1c, hemoglobin A1c; HDL-C, high-density lipoprotein cholesterol; LDL-C: low-density lipoprotein cholesterol; ApoA I, apolipoprotein A I; and ApoB, apolipoprotein B.

and RHI was found for the patients without statins or antihypertensive medication compared with those with these medications. A $0.1 \mathrm{mmHg} /$ minute increase in daytime rate of SBP variation was associated with a 0.42 decrease in RHI in patients without statin use, -0.23 for patients without $\beta$-blocker, -0.32 for patients without ACEI/ARB use, and -0.28 for patients without diuretics (all $P<0.05$ ). We also found taking $\mathrm{CCB}$ or acarbose did not affect the results. For the subjects with $\mathrm{eGFR} \geq 60 \mathrm{~mL} /\left(\right.$ minute* $\left.1.73 \mathrm{~m}^{2}\right)$, we observed that a 0.17 decrease in RHI was associated with a per $0.1 \mathrm{mmHg} / \mathrm{minute}$ increase in daytime rate of SBP variation (Table VI). Table VII indicates that a greater decrease in RHI was associated with a $0.1 \mathrm{mmHg} /$ minute increase in daytime rate of SBP variation in patients with diabetes $(n=16)(\beta=-0.860, P=0.001)$.

In simple or multiple regression models, no significant 
Table II. Ambulatory BP Parameters of All Subjects

\begin{tabular}{|c|c|}
\hline ABPM parameter & Mean (SD) \\
\hline 24-hour SBP (mmHg) & $124.1(10.3)$ \\
\hline 24-hour DBP (mmHg) & $75.5(7.5)$ \\
\hline 24-hour heart rate (bpm) & $69.0(9.2)$ \\
\hline Daytime SBP (mmHg) & $125.9(10.3)$ \\
\hline Daytime DBP (mmHg) & $77.0(7.8)$ \\
\hline Nighttime SBP (mmHg) & $119.1(11.7)$ \\
\hline Nighttime DBP (mmHg) & $71.7(7.8)$ \\
\hline 24-hour rate of SBP variation ( $\mathrm{mmHg} /$ minute) & $0.388(0.073)$ \\
\hline 24-hour rate of $\mathrm{DBP}$ variation (mmHg/minute) & $0.317(0.064)$ \\
\hline Daytime rate of SBP variation (mmHg/minute) & $0.431(0.089)$ \\
\hline Daytime rate of DBP variation ( $\mathrm{mmHg} /$ minute) & $0.351(0.095)$ \\
\hline Nighttime rate of SBP variation (mmHg/minute) & $0.273(0.082)$ \\
\hline Nighttime rate of DBP variation (mmHg/minute) & $0.239(0.064)$ \\
\hline Average rate of SBP variation during morning $\mathrm{BP}$ surge ( $\mathrm{mmHg} / \mathrm{minute})^{*}$ & $0.459(0.154)$ \\
\hline Average rate of DBP variation during morning $\mathrm{BP}$ surge (mmHg/minute) & $0.358(0.123)$ \\
\hline SD of 24-hour SBP (mmHg) & $11.7(2.4)$ \\
\hline SD of 24-hour DBP (mmHg) & $9.3(1.7)$ \\
\hline SD of daytime SBP (mmHg) & $11.3(2.4)$ \\
\hline SD of daytime DBP (mmHg) & $8.8(2.1)$ \\
\hline SD of nighttime SBP (mmHg) & $10.0(3.0)$ \\
\hline SD of nighttime DBP (mmHg) & $8.6(2.1)$ \\
\hline SD of SBP during morning surge $(\mathrm{mmHg})$ & $10.1(2.9)$ \\
\hline SD of DBP during morning surge (mmHg) & $7.8(2.5)$ \\
\hline CV of 24-hour SBP (\%) & $9.5(2.0)$ \\
\hline CV of 24-hour DBP (\%) & $12.4(2.4)$ \\
\hline CV of daytime SBP (\%) & $9.0(2.0)$ \\
\hline CV of daytime DBP (\%) & $11.6(2.9)$ \\
\hline CV of nighttime SBP (\%) & $8.5(2.7)$ \\
\hline CV of nighttime DBP (\%) & $12.0(3.1)$ \\
\hline CV of SBP during morning surge (\%) & $8.0(2.4)$ \\
\hline $\mathrm{CV}$ of DBP during morning surge $(\%)$ & $10.0(3.2)$ \\
\hline
\end{tabular}

SBP indicates systolic blood pressure; DBP, diastolic blood pressure; and CV, coefficient of variation. *Average rate of BP variation during the morning BP surge was computed for the time interval of 6:00 am to 10:00 am.

association was found between RHI and PWA parameters.

\section{Discussion}

A group of patients with MetS was enrolled in this crosssectional study whose aim was to evaluate the association between time rate of BP variation and RHI, an index reflecting microvascular endothelial function. The results indicate that the daytime rate of SBP variation was negatively associated with RHI independent of BP level and central hemodynamics. A $0.1 \mathrm{mmHg} /$ minute increase in the daytime rate of SBP variation correlated to a decrease of 0.20 in RHI. RHI evaluated by Endo-PAT reflects a nitric oxide (NO) mediated response, which has been confirmed by Nohria, et al at Brigham \& Women's Hospital, Boston. ${ }^{15)}$ In addition, more evidence has been suggested that lower endothelial function measured by PAT was associated with several traditional cardio-metabolic and cardiovascular risk factors, and as a predictor of cardiovascular outcome. ${ }^{16)}$

Hodgson, et al in 2014 concluded that the rate of BP variation was negatively associated with flow-mediated dilation (FMD) and glyceryl trinitrate (GTN)-mediated dilation, both of which were measures of endothelial and smooth muscle cell function, and relationships were attenuated after accounting for age and BP in a cross-sectional study of a group of treated hypertensive and untreated largely normotensive subjects. ${ }^{17)}$
Diaz, et al conducted a small cross-sectional study $(n=36)$, which explored the relationships of time rate of BP variation with endothelium-dependent and independent brachial artery vasodilation. Consistent with the present study, the daytime rate of SBP variation may be considered as an indicator of endothelial function, independent of BP. ${ }^{18)}$ Current evidence confirms the idea that higher short-term BP variation contributes to target organ damage and cardiovascular diseases risk. ${ }^{19,20)}$ Vascular endothelial and smooth muscle cells are clear targets for the damage.

The present study results were also consistent with current evidence on the association between the time rate of BP variation and target-organ damage. Zakopoulos, et al reported that the 24-hour rate of SBP variation and the rate of SBP variation during the morning $\mathrm{BP}$ surge correlated independently to larger common carotid artery (CCA) intima-media thickness (IMT) values, which indicates that steeper BP changes may lead to a greater stress on the vessel wall and further to medial hypertrophy of the large arteries. ${ }^{4}$ In addition, a $0.1 \mathrm{mmHg} /$ minute increase in the daytime rate of SBP variation was correlated to an increment of 4.935 in the Gensini score, an index assessing the topography and severity of coronary artery lesions, which was reported by Manios, et al in 2011. ${ }^{8)}$ The clear association of time rate with endothelial function and CCAIMT, which are the early stages in the process of atherosclerosis, suggests that the time rate of BP variation may become the best indicator of early atherosclerosis. Moreover, the 24-hour 
Table III. Simple Linear Regression Analyses of Variables Associated With RHI

\begin{tabular}{|c|c|c|c|}
\hline Variable & $\begin{array}{c}\text { General } \\
\text { Regression } \\
\text { Coefficients (se) }\end{array}$ & $\begin{array}{l}\text { Standardized } \\
\text { Coefficients }\end{array}$ & $P$ \\
\hline Age (years) & $-0.016(0.007)$ & -0.272 & 0.035 \\
\hline Gender & $0.007(0.138)$ & 0.007 & 0.958 \\
\hline Diabetes & $-0.118(0.158)$ & -0.098 & 0.456 \\
\hline Hypertension & $0.061(0.184)$ & 0.043 & 0.742 \\
\hline Dyslipidemia & $-0.012(0.162)$ & -0.009 & 0.943 \\
\hline Statins & $-0.314(0.132)$ & -0.299 & 0.020 \\
\hline$\beta$-Blocker & $-0.119(0.143)$ & 0.108 & 0.411 \\
\hline Calcium channel blocker & $0.087(0.137)$ & 0.083 & 0.527 \\
\hline Angiotensin-converting enzyme inhibitor & $-0.126(0.275)$ & 0.060 & 0.649 \\
\hline Angiotensin receptor blocker & $0.188(0.139)$ & 0.175 & 0.180 \\
\hline Diuretics & $0.080(0.171)$ & 0.061 & 0.644 \\
\hline $\operatorname{BMI}\left(\mathrm{kg} / \mathrm{m}^{2}\right)$ & $0.037(0.025)$ & 0.189 & 0.147 \\
\hline Glucose (mmol/L) & $-0.086(0.040)$ & -0.270 & 0.037 \\
\hline $\operatorname{HbA1c}(\%)$ & $-0.117(0.067)$ & -0.224 & 0.085 \\
\hline Total cholesterol (mmol/L) & $0.087(0.060)$ & 0.187 & 0.152 \\
\hline Triglycerides (mmol/L) & $0.158(0.086)$ & 0.234 & 0.072 \\
\hline $\mathrm{HDL}-\mathrm{C}(\mathrm{mmol} / \mathrm{L})$ & $-0.130(0.266)$ & -0.064 & 0.626 \\
\hline LDL-C (mmol/L) & $0.132(0.086)$ & 0.197 & 0.131 \\
\hline eGFR (CKD-EPI) & $0.002(0.002)$ & 0.135 & 0.303 \\
\hline \multicolumn{4}{|l|}{ ABPM parameters } \\
\hline 24-hour mean SBP (mmHg) & $-0.006(0.007)$ & -0.121 & 0.358 \\
\hline 24-hour mean DBP (mmHg) & $0.011(0.009)$ & 0.151 & 0.250 \\
\hline 24-hour mean HR (bpm) & $0.005(0.007)$ & 0.089 & 0.501 \\
\hline Daytime mean SBP (mmHg) & $-0.004(0.007)$ & -0.087 & 0.509 \\
\hline Daytime mean DBP (mmHg) & $0.013(0.009)$ & 0.009 & 0.129 \\
\hline Daytime mean HR (bpm) & $0.005(0.007)$ & 0.098 & 0.455 \\
\hline Nighttime mean SBP (mmHg) & $-0.008(0.006)$ & -0.167 & 0.202 \\
\hline Nighttime mean DBP (mmHg) & $0.004(0.009)$ & 0.063 & 0.631 \\
\hline Nighttime mean HR (bpm) & $0.004(0.008)$ & 0.059 & 0.654 \\
\hline Mean SBP during morning surge $(\mathrm{mmHg})$ & $-0.001(0.005)$ & -0.016 & 0.902 \\
\hline Mean DBP during morning surge (mmHg) & $0.009(0.008)$ & 0.156 & 0.234 \\
\hline 24-hour rate of SBP variation (mmHg/minute) & $-2.176(0.901)$ & -0.302 & 0.019 \\
\hline 24-hour rate of DBP variation (mmHg/minute) & $-0.947(1.074)$ & -0.115 & 0.382 \\
\hline Daytime rate of SBP variation (mmHg/minute) & $-1.914(0.736)$ & -0.323 & 0.012 \\
\hline Daytime rate of DBP variation (mmHg/minute) & $-0.993(0.718)$ & -0.179 & 0.172 \\
\hline Nighttime rate of SBP variation ( $\mathrm{mmHg} /$ minute) & $-0.008(0.843)$ & -0.001 & 0.993 \\
\hline Nighttime rate of DBP variation ( $\mathrm{mmHg} /$ minute) & $0.716(1.062)$ & 0.088 & 0.503 \\
\hline Rate of SBP variation during morning $\mathrm{BP}$ surge (mmHg/minute) & $-0.977(0.441)$ & -0.279 & 0.031 \\
\hline Rate of DBP variation during morning $\mathrm{BP}$ surge ( $\mathrm{mmHg} /$ minute) & $-0.779(0.548)$ & -0.183 & 0.161 \\
\hline $\mathrm{SD}$ of 24-hour mean SBP $(\mathrm{mmHg})$ & $-0.037(0.029)$ & -0.164 & 0.212 \\
\hline SD of 24-hour mean DBP (mmHg) & $0.010(0.040)$ & 0.031 & 0.812 \\
\hline SD of daytime $\mathrm{SBP}(\mathrm{mmHg})$ & $-0.055(0.029)$ & -0.244 & 0.060 \\
\hline SD of daytime $\mathrm{DBP}(\mathrm{mmHg})$ & $-0.004(0.034)$ & -0.016 & 0.906 \\
\hline SD of nighttime SBP $(\mathrm{mmHg})$ & $-0.005(0.023)$ & -0.028 & 0.831 \\
\hline SD of nighttime DBP (mmHg) & $-0.002(0.032)$ & -0.008 & 0.952 \\
\hline SD of SBP during morning surge (mmHg) & $-0.033(0.024)$ & -0.177 & 0.177 \\
\hline SD of DBP during morning surge (mmHg) & $-0.010(0.028)$ & -0.046 & 0.724 \\
\hline $\mathrm{CV}$ of 24-hour mean SBP $(\%)$ & $-0.031(0.036)$ & -0.114 & 0.387 \\
\hline CV of 24-hour mean DBP $(\%)$ & $-0.009(0.029)$ & -0.041 & 0.757 \\
\hline CV of daytime SBP (\%) & $-0.062(0.036)$ & -0.219 & 0.093 \\
\hline CV of daytime DBP (\%) & $-0.021(0.024)$ & -0.112 & 0.394 \\
\hline CV of nighttime SBP $(\%)$ & $0.003(0.025)$ & 0.014 & 0.917 \\
\hline $\mathrm{CV}$ of nighttime $\mathrm{DBP}(\%)$ & $-0.005(0.022)$ & -0.031 & 0.812 \\
\hline CV of SBP during morning surge (\%) & $-0.038(0.030)$ & -0.166 & 0.205 \\
\hline $\mathrm{CV}$ of DBP during morning surge (\%) & $-0.018(0.022)$ & -0.109 & 0.407 \\
\hline \multicolumn{4}{|l|}{ PWA parameters } \\
\hline Aortic SBP (mmHg) & $0.007(0.005)$ & 0.188 & 0.151 \\
\hline Aortic DBP (mmHg) & $0.005(0.007)$ & 0.103 & 0.434 \\
\hline Aortic pulse pressure (mmHg) & $0.006(0.006)$ & 0.132 & 0.313 \\
\hline Augmentation pressure (mmHg) & $0.015(0.010)$ & 0.182 & 0.163 \\
\hline Augmentation index & $0.009(0.008)$ & 0.148 & 0.259 \\
\hline
\end{tabular}

BMI indicates body mass index; HbAlc, hemoglobin Alc; HDL-C, high-density lipoprotein cholesterol; LDL-C, low-density lipoprotein cholesterol; SBP, systolic blood pressure; DBP, diastolic blood pressure; and CV, coefficient of variation. 
Table IV. Factors Associated Independently With RHI in the Multiple Regression Analyses With Stepwise Procedure

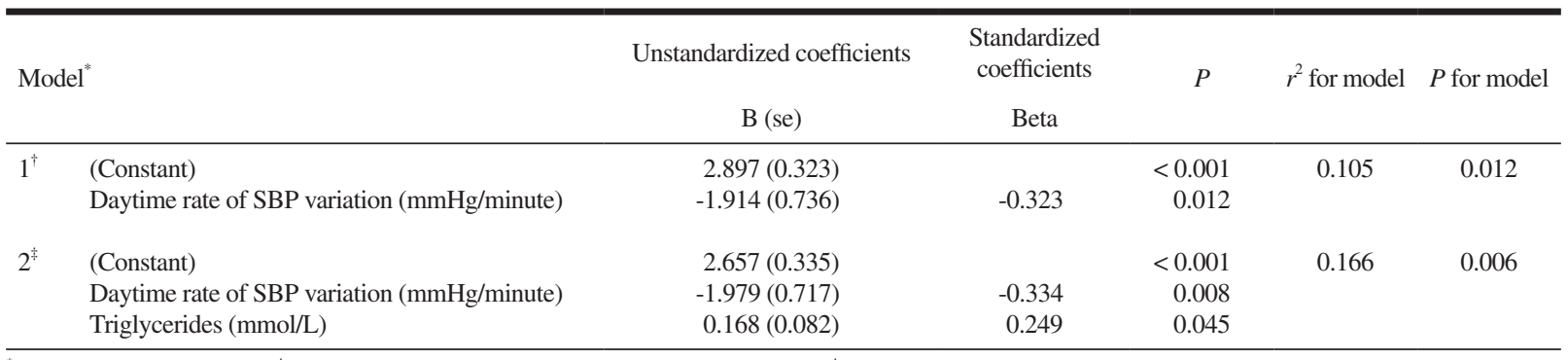

"Dependent variable: RHI. 'Predictors: (Constant), Daytime rate of SBP variation. "Predictors: (Constant), Daytime rate of SBP variation, Triglycerides.

Table V. Significant Association of Factors With RHI Between the Groups With or Without Pharmacological Interventions After Controlling Confounders

\begin{tabular}{|c|c|c|c|c|}
\hline \multirow{2}{*}{ Subgroup } & & \multicolumn{3}{|c|}{ Daytime rate of SBP variation } \\
\hline & & $\mathrm{B}(\mathrm{se})$ & $\beta$ & $P$ \\
\hline \multirow[t]{2}{*}{ Statin } & Yes $(n=34)$ & $-0.695(0.795)$ & -0.155 & 0.389 \\
\hline & No $(n=27)$ & $-4.154(1.468)$ & -0.493 & 0.009 \\
\hline \multirow[t]{2}{*}{$\beta$-Blocker } & Yes $(n=22)$ & $-1.910(1.408)$ & -0.297 & 0.191 \\
\hline & No $(n=39)$ & $-2.281(0.896)$ & -0.386 & 0.015 \\
\hline \multirow[t]{2}{*}{ Calcium channel blocker } & Yes $(n=32)$ & $-1.486(1.060)$ & -0.247 & 0.172 \\
\hline & No $(n=29)$ & $-1.824(1.062)$ & -0.314 & 0.097 \\
\hline \multirow[t]{2}{*}{ Angiotensin-converting enzyme inhibitor/ Angiotensin receptor blocker } & Yes $(n=27)$ & $-0.783(1.058)$ & -0.150 & 0.466 \\
\hline & No $(n=34)$ & $-3.160(1.103)$ & -0.458 & 0.007 \\
\hline \multirow[t]{2}{*}{ Diuretic } & Yes $(n=12)$ & $1.588(0.714)$ & 0.381 & 0.057 \\
\hline & No $(n=49)$ & $-2.798(0.916)$ & -0.411 & 0.004 \\
\hline \multirow[t]{2}{*}{ Acarbose } & Yes $(n=16)$ & $-2.655(1.227)$ & -0.465 & 0.051 \\
\hline & No $(n=45)$ & $-0.866(0.893)$ & -0.145 & 0.338 \\
\hline
\end{tabular}

Dependent variable: RHI. The analyses were adjusted by gender, age, BMI, disease status, 24-hour BP, total cholesterol, triglycerides, and other medications.

Table VI. Sensitive Analysis of the Association Between RHI and Daytime Rate of SBP Variation For the Patients With eGFR $\geq 60 \mathrm{~mL} /\left(\mathrm{minute} * 1.73 \mathrm{~m}{ }^{2}\right)$

\begin{tabular}{lcccc}
\hline Model & $\begin{array}{c}\text { Unstandardized } \\
\text { Coefficients }\end{array}$ & $\begin{array}{c}\text { Standardized } \\
\text { Coefficients }\end{array}$ & $P$ & $r^{2}$ for model \\
\hline Daytime rate of SBP variation $(\mathrm{mmHg} /$ minute $)$ & $\mathrm{B}(\mathrm{se})$ & Beta & & \\
\hline
\end{tabular}

Dependent Variable: RHI. The analyses were adjusted by gender, age, BMI, disease status, 24-hour BP, total cholesterol, triglycerides, and other medications.

Table VII. Sensitive Analysis of the Association Between RHI and Daytime Rate of SBP Variation For the Patients With or Without Diabetes After Controlling Confounders

\begin{tabular}{|c|c|c|c|c|c|c|}
\hline \multirow{2}{*}{ Regression analyses } & \multicolumn{3}{|c|}{ Group with diabetes } & \multicolumn{3}{|c|}{ Group without diabetes } \\
\hline & $\mathrm{B}(\mathrm{se})$ & $\beta$ & $P$ & $\mathrm{~B}(\mathrm{se})$ & $\beta$ & $P$ \\
\hline Daytime rate of SBP variation ( $\mathrm{mmHg} /$ minute) & $-2.655(1.227)$ & -0.465 & 0.051 & $-0.866(0.893)$ & -0.145 & 0.338 \\
\hline
\end{tabular}

Dependent variable: RHI. The analyses were adjusted by gender, age, BMI, disease status, 24-hour BP, total cholesterol, triglycerides, and other medications. 
rate of SBP variation is independently associated with impaired renal function and brain edema, with an odds ratio (OR) of 1.49 and 1.139 associated with a $0.1 \mathrm{mmHg} /$ minute increase in the 24-hour rate of SBP variation, respectively. ${ }^{8,21)}$

We also conclude there was a greater association for those who were not taking statins. Mihos, et al reviewed the current evidence of the pleiotropic effects of statins, which include the enhancement of endothelial function and an antihypertensive effect. ${ }^{22)}$ Therefore, these two pathways may partly explain the interference effect of statins on the association between the time rate of BP variation and RHI. As well as the greater association for those without intake of $\beta$-blockers, $\mathrm{ACEI} / \mathrm{ARB}$, or diuretics, antihypertensive drugs may change the BPV in patients with hypertension, which may result in the change of association. However, we must be careful making this conclusion due to the small sample size. Further verification is needed in a larger sample size. In addition, diabetes was a modified factor based on our study. Consistent with the results from the Action in Diabetes and Vascular Disease (ADVANCE) Trial, ${ }^{23)}$ which included 8811 patients, visit-to-visit variability of SBP clearly predicted the future development of major microvascular complications among patients with diabetes.

It is well known that an increasing BP variability is associated with the development, progression, and severity of cardiovascular and renal organ damage and with an increased risk of cardiovascular events and mortality. ${ }^{24)}$ However, an association of BP variability indices, quantified as SD and CV of BP, with RHI was not found in our multiple regression analyses. Therefore, the results indicate that endothelial dysfunction is correlated with an increased daytime rate of SBP variation, which focuses on the subsequent changes between consecutive $\mathrm{BP}$ recordings and is more sensitive than absolute changes of $\mathrm{BP}$ recordings.

There are several possible mechanisms for the association between daytime rate of SBP variation and endothelial function. Hemodynamic shear stress, the frictional force acting on vascular endothelial cells, is crucial for endothelial homeostasis under normal physiological conditions. There are two patterns of blood flow: steady laminar flow and disturbed flow. Laminar flow which exerts steady laminar shear stress is atheroprotective while disturbed flow creates an atheroprone environment, which not only correlates with different shear stress in the vessel walls, but also with a different response of endothelial cells. Emerging evidence has provided new insights into the cellular mechanisms of flow-dependent regulation of vascular function that leads to cardiovascular events such as atherosclerosis, atherothrombosis, and myocardial infarction. ${ }^{25)}$ Endothelial dysfunction caused by disturbed flow is thought to be one of the primary culprits in the progression of atherosclerosis. Time rate of BP variation, as an index of shear stress in the vessel wall, may pose a great threat to endothelial cells in certain signal pathways, including inflammation and oxidative stress, and further promote platelet aggregation and plaque formation. In addition, the continuous exposure of endothelial cells to oscillatory shear stress has been suggested to cause the activation of a pro-oxidant process, leading to redoxsensitive expression of endothelial proinflammatory genes. ${ }^{26)}$

There are several limitations in the present study. First, the time rate of BP variation was calculated by ambulatory BP measured every 20 minutes during the day and every 30 min- utes during the night, which was not as good as invasive intraarterial BP monitoring in reflecting short-lasting BP fluctuations. However, ambulatory BP monitoring has several advantages, such as being non-invasive, simple, and convenient. Most importantly, it can monitor BP for 24 hours, covering the daytime and nighttime. Second, the small sample size is a major limitation, which may lead to the poor generalizability of our results. Third, it should be acknowledged that the cross-sectional design of the present study cannot determine whether an increased rate of BP changes can promote endothelial dysfunction. Fourth, to a large extent, the time rate of BP variation is determined by the magnitude of BP within a certain time period when the interval of BP measurement by ABPM is fixed. One may argue that there is theoretically no significant implication of the rate of BP variation when compared with the actual BP variability in the study setting of intermittent ambulatory BP measurements. However, the rate of $\mathrm{BP}$ variation was the only ABPM parameter that was retained as an independent determinant of RHI in the multiple regression models.

In conclusion, our study findings indicate that the daytime rate of SBP variation is independently associated with endothelial function, which is one type of target-organ damage. The negative association of daytime rate of SBP variation with endothelial function is independent of BP level, BP variability, and central hemodynamics. The association may be greater for those without antihypertensive medication or statins. Further research is needed to verify the association between both and to determine whether the higher time rate of $\mathrm{BP}$ variation will result in endothelial dysfunction.

\section{Disclosure}

None.

\section{ACKNOWLedgments}

The determination of biochemical indicators was supported by Lu Han of the Clinical Laboratory of Peking Union Medical College Hospital. The authors wish to thank the subjects for participating in the study.

\section{REFERENCES}

1. Hermida RC, Ayala DE, Mojón A, Fernández JR, Smolensky M, Portaluppi F. Morning surge, dipping, and sleep-time blood pressure as prognostic markers of cardiovascular risk. Hypertension 2013; 61: e3.

2. Wang C, Zhang J, Liu X, et al. Reversed dipper blood-pressure pattern is closely related to severe renal and cardiovascular damage in patients with chronic kidney disease. PLoS One 2013; 8: e55419.

3. Mancia G, Parati G, Castiqlioni P, et al. Daily life blood pressure changes are steeper in hypertensive than in normotensive subjects. Hypertension 2003; 42: 277-82.

4. Zakopoulos NA, Tsivqoulis G, Barlas G, et al. Time rate of blood pressure variation is associated with increased common carotid artery intima-media thickness. Hypertension 2005; 45: 505-12.

5. Stamatelopoulos KS, Manios E, Barlas G, et al. Time rate of blood pressure variation is superior to central hemodynamics as an asso- 
ciate of carotid intima-media thickness. J Hypertens 2010; 28: 518.

6. Zakopoulos NA, Tsivgoulis G, Barlas G, et al. Impact of the time rate of blood pressure variation on left ventricular mass. J Hypertens 2006; 24: 2071-7.

7. Manios E, Tsagalis G, Tsivgoulis G, et al. Time rate of blood pressure variation is associated with impaired renal function in hypertensive patients. J Hypertens 2009; 27: 2244-8.

8. Manios E, Stamatelopoulos K, Tsivgoulis G, et al. Time rate of blood pressure variation: a new factor associated with coronary atherosclerosis. J Hypertens 2011; 29: 1109-14.

9. Lerman A, Burnett JC Jr. Intact and altered endothelium in regulation of vasomotor. Circulation 1992; 86: III12-9. (Review)

10. Bonetti PO, Lerman LO, Lerman A. Endothelial dysfunction: a marker of atherosclerotic risk. Arterioscler Thromb Vasc Biol 2003; 23: 168-75. (Review)

11. Kuvin JT, Patel AR, Sliney KA, et al. Assessment of peripheral vascular endothelial function with finger arterial pulse wave amplitude. Am Heart J 2003; 146: 168-74.

12. Hamburg NM, Keyes MJ, Larson MG, et al. Cross-sectional relations of digital vascular function to cardiovascular risk factors in the Framingham Heart Study. Circulation 2008; 117: 2467-74.

13. Malek AM, Alper SL, Izumo S. Hemodynamic shear stress and its role in atherosclerosis. JAMA 1999; 282: 2035-42.

14. Williams B, Lacy PS, Thom SM, et al. Differential impact of blood pressure-lowering drugs on central aortic pressure and clinical outcomes: principal results of the Conduit Artery Function Evaluation (CAFE) study. Circulation 2006; 113: 1213-25.

15. Nohria A, Gerhard-Herman M, Creager MA, Hurley S, Mitra D, Ganz P. Role of nitric oxide in the regulation of digital pulse volume amplitude in humans. J Appl Physiol 2006; 101: 545-8.

16. Suessenbacher A, Dörler J, Wanitschek M, F Alber H, Pachinger O, Frick M. Prognostic value of peripheral arterial tonometry in patients with coronary artery disease and a high cardiovascular risk profile. J Atheroscler Thromb 2014; 21: 230-8.

17. Hodgson JM, Woodman RJ, Croft KD, et al. Relationships of vascular function with measures of ambulatory blood pressure variation. Atherosclerosis 2014; 233: 48-54.
18. Diaz KM, Veerabhadrappa P, Kashem MA, et al. Relationship of visit-to-visit and ambulatory blood pressure variability to vascular function in African Americans. Hypertens Res 2012; 35: 55-61.

19. Eguchi K, Ishikawa J, Hoshide S, et al. Night time blood pressure variability is a strong predictor for cardiovascular events in patients with type 2 diabetes. Am J Hypertens 2009; 22: 46-51.

20. Rothwell PM. Limitations of the usual blood-pressure hypothesis and importance of variability, instability, and episodic hypertension. Lancet 2010; 375: 938-48. (Review)

21. Skalidi SJ, Manios ED, Stamatelopoulos KS, et al. Brain edema formation is associated with the time rate of blood pressure variation in acute stroke patients. Blood Press Monit 2013; 18: 203-7.

22. Mihos CG, Pineda AM, Santana O. Cardiovascular effects of statins, beyond lipid-lowering properties. Pharmacol Res 2014; 88: 12-9. (Review)

23. Hata J, Arima H, Rothwell PM, et al. Effects of visit-to-visit variability in systolic blood pressure on macrovascular and microvascular complications in patients with type 2 diabetes mellitus: the ADVANCE trial. Circulation 2013; 128: 1325-34.

24. Parati G, Ochoa JE, Lombardi C, Salvi P, Bilo G. Assessment and interpretation of blood pressure variability in a clinical setting. Blood Press 2013; 22: 345-54. (Review)

25. Heo KS, Fujiwara K, Abe J. Shear stress and atherosclerosis. Mol Cells 2014; 37: 435-40. (Review)

26. De Keulenaer GW, Chappell DC, Ishikaza N, Nerem RM, Alexander RW, Griendling KK. Oscillatory and steady laminar shear stress differentially affect human endothelial redox state: role of a superoxide-producing NADH oxidase. Circ Res 1998; 82: 1094101

\section{SuPPLEMENTAL File}

Supplemental Table I, II, III, IV, V

Please find supplemental file;

https://www.jstage.jst.co.jp/article/ihj/57/2/57_15-322/_article/supplement 